\title{
Retrospective analysis of 14 cases of disseminated Penicillium marneffei infection with osteolytic lesions
}

Ye Qiu ${ }^{1+}$, Jianquan Zhang ${ }^{2^{*}{ }^{+}}$, Guangnan Liu' ${ }^{2}$ Xiaoning Zhong ${ }^{2}$, Jingmin Deng ${ }^{2}$, Zhiyi He ${ }^{2}$ and Bai Jing ${ }^{2}$

\begin{abstract}
Background: Penicillium marneffei disseminates hematogenously and can infect most organs, though infection leading to osteolysis is extremely rare. We describe the clinical and laboratory features, management, and outcomes of patients with penicilliosis marneffei (PSM) with osteolytic lesions.

Methods: This retrospective study was conducted between January 1, 2003 and May 1, 2014 at the First Affiliated Hospital of Guangxi Medical University. Patients who presented with culture and/or histopathologic proof of disseminated PSM within osteolytic lesions were included.

Results: P. marneffei infection was diagnosed in 100 patients (65 HIV-infected and 35 HIV-negative). Fourteen patients, all HIV-negative, $(14 / 35,40 \%)$ had osteolytic lesions. The most common comorbidity was diabetes mellitus, though previous glucocorticoid therapy, $\beta$-thalassemia, breast cancer, and Langerhans cell histiocytosis also occurred. Five patients had no comorbidity. Fever, malaise, ostealgia, weight loss, and anemia were the most common symptoms, followed by cutaneous lesions, lymphadenopathy, hepatosplenomegaly, cough, sputum, and stethalgia. Ostealgia, joint pain, and joint disorders were also recorded. White blood cell and neutrophil counts were increased (mean $22.3 \pm 7.4 \times 10^{9}$ cells $/ L$; mean $18.84 \pm 4.5 \times 10^{9}$ cells/L, respectively). The most common sites were the vertebrae, skull and femur, ribs and ilium, though the clavicle, scapula, humerus, and tibia were also involved. Radiography and computed tomography $(C T)$ showed multiple radiolucencies with moth-eaten bone destruction, periosteal proliferation, bone fracture, and surrounding soft-tissue swelling. Emission $C T$ showed significantly increased uptake in many skeletal regions. Positron emission tomography/CT showed generalized lymphadenopathy, bone metabolic activity, and bone destruction. The ${ }^{18}$ F-FDG standard uptake value was increased in the entire skeleton (mean 6.16). Twelve patients received antifungal therapy, four of whom died during treatment, and eight recovered, though four of these eight relapsed within 3-24 months. Two patients discontinued treatment because of severe multiple organ failure and died.
\end{abstract}

Conclusions: Osteolysis is often overlooked in HIV-negative individuals with disseminated P. marneffei infection. However, P. marneffei involving the bone and leading to osteolysis may indicate severe systemic disturbance, and is characterized by a poor prognosis, high recurrence rate, and the need for prolonged antifungal treatment.

Keywords: HIV-negative, Penicilliosis marneffei, Ostealgia, Osteolytic lesion

\footnotetext{
* Correspondence: jazhang2002@sina.com

'Equal contributors

${ }^{2}$ Department of Respiratory Medicine, The First Affiliated Hospital of Guangxi

Medical University, Nanning, Guangxi 530021, China

Full list of author information is available at the end of the article
} 


\section{Background}

Penicilliosis marneffei (PSM) is a deep fungal infection caused by Penicillium marneffei, and one of the most common opportunistic infections among patients with AIDS in Southeast Asia [1]. PSM is categorized as either localized or disseminated. Disseminated PSM usually affects the monocyte-macrophage system in multiple organs including the skin, lungs, and the reticuloendothelial system [2], while P. marneffei infection of the bone leading to osteolytic lesions is extremely rare [3]. In this study, we retrospectively analyzed the medical records and clinical characteristics of 14 patients diagnosed with disseminated PSM with osteolytic lesions between January 1, 2003 and May 1, 2014, at the First Affiliated Hospital of Guangxi Medical University. We describe the clinical and laboratory features, management, and outcomes of these patients to provide evidence for the correct diagnosis and early treatment of this condition.

\section{Methods}

\section{Medical records}

The medical records of 100 patients diagnosed with PSM between January 1, 2003 and May 1, 2014, at the First Affiliated Hospital of Guangxi Medical University were reviewed. Among these, 14 patients with osteolytic lesions were retrospectively evaluated. Patient information, including demographics (sex, age, and occupation), medical history (present history, comorbidities, and previous therapy), auxiliary examination results (hematological tests, serological tests, immune status, imaging examinations, and pathological and microbiological tests), and treatment, was collected from medical records and summarized for analysis.

This study was approved by the First Affiliated Hospital of Guangxi Medical University Signation Ethical Committee. All patients provided written informed consent.

\section{Diagnostic criteria for disseminated PSM}

$P$. marneffei was isolated from clinical specimens including blood, bone marrow, bone, sputum, lung, or discharge from skin lesions and cultured on Sabouraud dextrose agar (SDA) at $25^{\circ} \mathrm{C}$ and $37^{\circ} \mathrm{C}$. Positive $P$. marneffei cultures were characterized by dimorphic fungi that grew as a mold at $25^{\circ} \mathrm{C}$ and as a yeast at $37^{\circ} \mathrm{C}$ [4]. P. marneffei was identified based on cytology and histopathology from tissues and secretions stained with periodic acid-Schiff stain or Wright-Giemsa stain, by light microscopy. The yeast form of P. marneffei has a characteristic morphology including a transverse septum [5].

\section{Diagnostic criteria for PSM with osteolytic lesion}

Patients were diagnosed according to the following criteria: (i) P. marneffei identified in bone and/or bone marrow biopsy samples using histopathology, cytologic smears, and fungal culture; (ii) disseminated PSM with osteolytic lesions diagnosed based on the presence of osteolytic lesions on imaging examination, clinical symptoms including ostealgia, improvement after receiving antifungal treatment alone, and exclusion of other diseases that cause osteolysis (tuberculosis, cancer, hematological diseases, and other fungal infections such as African histoplasmosis, blastomycosis, cryptococcosis, and coccidioidomycosis).

\section{Results}

\section{Demographic data and clinical characteristics}

During the 11-year study period, 100 patients were diagnosed with disseminated PSM according to the inclusion criteria, including $65 \mathrm{HIV}$-infected and $35 \mathrm{HIV}$-negative patients. Of these, only 14 patients, all HIV-negative, had osteolytic lesions and were enrolled. The incidence of P. marneffei with osteolytic lesions in HIV-negative patients was therefore $40 \%(14 / 35)$. The study population included nine men and five women with a median age of 42.17 years (range 22-67 years). Their occupations included farmer $(n=8)$, laborer $(n=3)$, cadre $(n=2)$, and student $(\mathrm{n}=1)$. HIV-negativity was determined as negative for interferon- $\gamma$ autoantibody using repeated enzyme-linked immunosorbent assay (ELISA) testing. Comorbidities included diabetes mellitus $(n=4)$, previous glucocorticoid therapy $(n=2)$, $\beta$-thalassemia $(n=1)$, breast cancer $(n=1)$, Langerhans cell histiocytosis $(n=1)$, and no comorbidities $(n=5)$. Fever, malaise, ostealgia, weight loss, and anemia were the most frequent symptoms, followed by cutaneous lesions, lymphadenopathy, hepatosplenomegaly, cough, sputum, and stethalgia. Ostealgia, joint pain, and joint disorders were also present (Table 1).

Table 1 Clinical manifestations in patients infected with Penicillium marneffei with osteolysis $(n=14)$

\begin{tabular}{lll}
\hline Symptom/sign & Patients & \\
\cline { 2 - 3 } & No. & $\%$ \\
\hline Fever & 14 & 100 \\
Malaise & 14 & 100 \\
Weight loss & 13 & 92.85 \\
Anaemia & 13 & 92.85 \\
Ostealgia & 13 & 92.85 \\
Cutaneous lesions & 12 & 85.71 \\
Lymphadenopathy & 11 & 78.57 \\
Hepatosplenomegaly & 10 & 71.43 \\
Cough and sputum production & 10 & 71.43 \\
Stethalgia & 9 & 64.28 \\
Dyspnea & 5 & 35.71 \\
Hemoptysis & 2 & 14.28 \\
\hline
\end{tabular}




\section{Laboratory examination}

Complete blood count examinations revealed increased white blood cell levels in 12 patients (85.71\%) (mean $22.3 \pm 7.4 \times 10^{9}$ cells $/ \mathrm{L}$, range $13.3-35 \times 10^{9}$ cells $/ \mathrm{L}$ ). The hemoglobin concentration was decreased in 13 patients (92.85\%) (mean $81.71 \pm 3.2 \mathrm{~g} / \mathrm{L}$, range 65-116 g/L). Neutrophils were increased in 13 patients $(92.85 \%)$ (mean $18.84 \pm 4.5 \times 10^{9}$ cells $/ \mathrm{L}$, range $8.8-28.2 \times 10^{9}$ cells $\left./ \mathrm{L}\right)$. The CD4 and CD8 lymphocyte counts were determined in 12 patients by flow cytometry and were decreased in nine patients (75\%). Serum biochemical analysis revealed a mean serum albumin concentration of $20.1 \pm 3.2 \mathrm{~g} / \mathrm{L}$ below the normal range in 13 patients $(92.85 \%$, range $11.0-31.4 \mathrm{~g} / \mathrm{L})$. Six patients (42.85\%) showed kidney dysfunction. C-reactive protein concentration and erythrocyte sedimentation rate were increased in all patients.

\section{Chest radiography and computed tomography}

Chest computed tomography (CT) indicated pulmonary consolidation in three patients $(21.43 \%)$, cavities in five (35.7\%), disseminated inflammation in 12 (85.71\%), and interstitial disease involving the pleura in two (14.28\%). Ten $(71.43 \%)$ patients had mediastinal and/or/ hilar lymphadenopathy, and nine (64.28\%) had pleural inflammatory reaction and/or pleural effusion. Four (28.57\%) patients had pericardial effusion, and three (21.43\%) had seroperitoneum.

\section{Skeletal localization}

In the 14 patients, $P$. marneffei infection involved multiple bony structures as follows: bones of the trunk $(n=11$, $78.57 \%)$, limbs $(n=10,71.43 \%)$, skull $(n=6,42.85 \%)$, and cartilage $(n=1,7.14 \%)$. The most common sites were the vertebrae $(n=10,71.43 \%)$, skull and femur $(n=6,42.85 \%)$, and ribs $(\mathrm{n}=5,35.71 \%)$. The ilium was affected in four (28.57\%) patients, the clavicle, scapula, humerus, and tibia in three (21.42\%), the radius, sternum, innominatum, fibula, and bones of the hand in two (14.28\%), and the elbow joint, ischium, pubis acetabulum, bones of the foot, and the sacrum were implicated in one $(7.14 \%)$ patient. Five (35.71\%) patients showed joint involvement. Soft-tissue inflammation was identified surrounding the bone in seven (50\%) patients (Table 2).

\section{Imaging examinations}

Radiographic and CT examinations showed multiple radiolucencies, resembling a moth-eaten pattern of bone destruction (Figure 1) in eight cases (57.14\%). Periosteal proliferation (Figure 2A) was identified in two cases (14.28\%), fracture (Figure 2A and B) in six cases (42.85\%), and surrounding soft-tissue swelling (Figure 1C) in five cases (35.71\%). Emission CT (ECT) was performed in five cases and showed significantly increased uptake in multiple bones (Figure 3). Positron emission tomography/CT
Table 2 Skeletal localization in patients infected with Penicillium marneffei with osteolysis $(n=14)$

\begin{tabular}{|c|c|c|c|}
\hline \multicolumn{2}{|c|}{ Types of bones and site } & \multicolumn{2}{|c|}{ Patients } \\
\hline & & \multirow{2}{*}{$\begin{array}{l}\text { No. } \\
42.85\end{array}$} & \multirow[t]{2}{*}{$\%$} \\
\hline Skull & 6 & & \\
\hline \multirow[t]{3}{*}{ Bones of the trunk } & Vertebrae & 10 & 71.43 \\
\hline & Ribs & 5 & 35.71 \\
\hline & Sternum & 2 & 14.28 \\
\hline \multirow[t]{16}{*}{ Bones of limbs } & Femur & 6 & 42.85 \\
\hline & Ilium & 4 & 28.57 \\
\hline & Clavicle & 3 & 21.42 \\
\hline & Scapula & 3 & 21.42 \\
\hline & Humerus & 3 & 21.42 \\
\hline & Shinbone & 3 & 21.42 \\
\hline & Radial, & 2 & 14.28 \\
\hline & Sternum, & 2 & 14.28 \\
\hline & Innominatum, & 2 & 14.28 \\
\hline & Fibula & 2 & 14.28 \\
\hline & Bones of hand & 2 & 14.28 \\
\hline & Elbow-bone & 1 & 7.14 \\
\hline & Ischium & 1 & 7.14 \\
\hline & Pubesacetabulum & 1 & 7.14 \\
\hline & Bones of foot & 1 & 7.14 \\
\hline & Sacrum & 1 & 7.14 \\
\hline Cartilage & 1 & 7.14 & \\
\hline Joint involvement & 5 & 35.71 & \\
\hline Soft tissue swelling & 7 & 50 & \\
\hline
\end{tabular}

(PET/CT) was performed in two cases and showed diffuse lymphadenopathy, whole-body bone metabolic activity, and destruction in multiple bones. The ${ }^{18}$ F-FDG standard uptake value (SUV) was increased in the entire skeleton (mean 6.16, range 2.5-9.6).

\section{Fungal culture and histopathology}

Fluid was aspirated from the bone marrow, blood, pleural effusion, seroperitoneum, subcutaneous abscess, skin ulcer, and dermal secretions, inoculated onto SDA, and incubated at $37^{\circ} \mathrm{C}$ and $25^{\circ} \mathrm{C}$. Twelve cases were confirmed by positive $P$. marneffei culture. P. marneffei was isolated from sputum samples $(1 / 14,7.14 \%)$, venous blood $(3 / 14$, $21.42 \%)$, bone marrow $(2 / 14,14.28 \%)$, bronchoalveolar lavage fluid $(1 / 14,7.14 \%)$, pus from a subcutaneous abscess $(3 / 14,21.42 \%)$ and dermal lesion secretions (3/14, 21.42\%). In addition, seven cases were diagnosed with $P$. marneffei infection by histopathology or cytology of specimens obtained from pulmonary $(1 / 14,7.14 \%)$, lymph node $(2 / 14$, $14.28 \%)$, bone marrow $(2 / 14,14.28 \%$, Figure $4 \mathrm{~A})$, bone $(1 / 14,7.14 \%$, Figure $4 B)$, and skin lesions $(2 / 14,14.28 \%)$. 

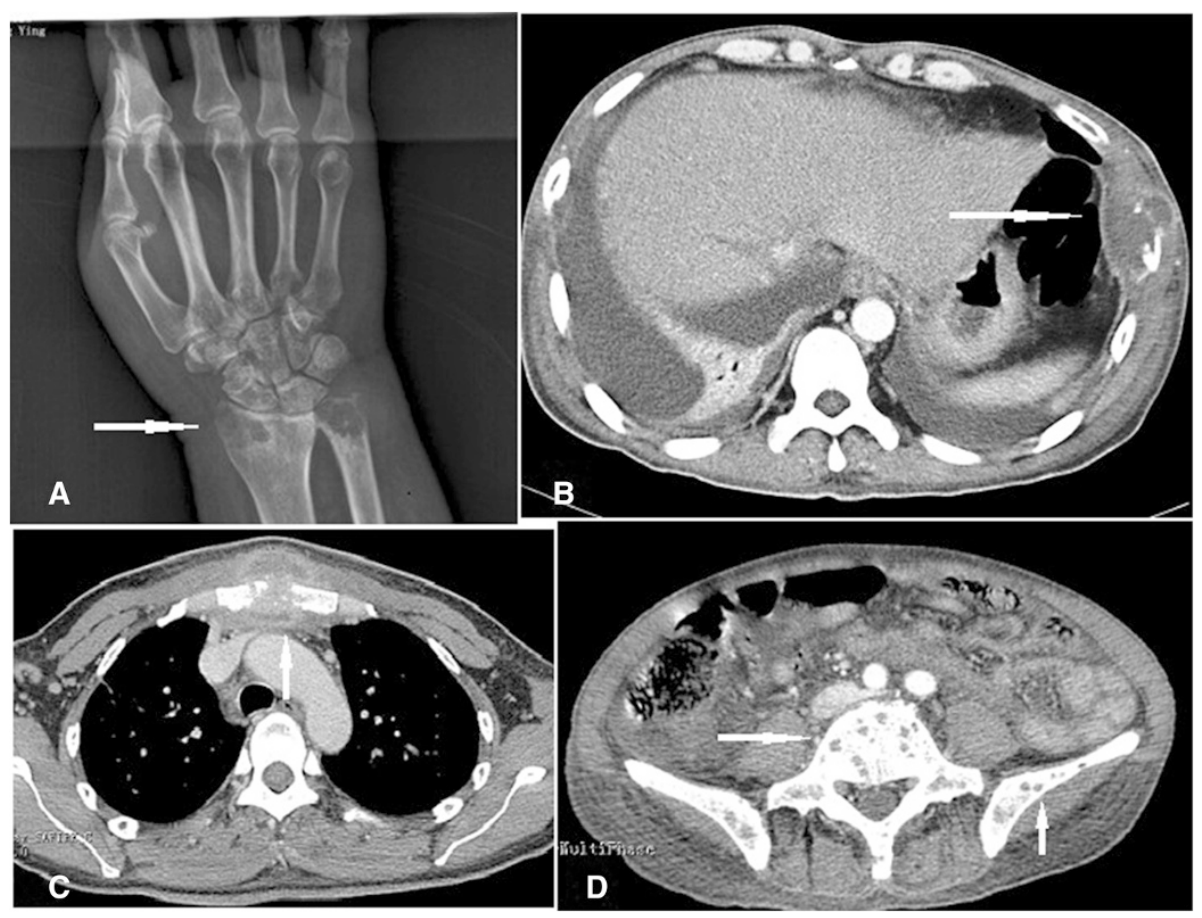

Figure 1 Radiography and computed tomography showing osteolytic lesions characterized by multiple lucent defects with a moth-eaten destruction of bone in the head of the radius (A), ulna (A), rib (B), sternum (C), vertebrae (D), and ilium (D), and swelling in surrounding soft tissues $(B, C)$.

\section{Treatment and outcome}

Twelve patients received intravenous amphotericin $\mathrm{B}$ deoxycholate $(0.6-1.0 \mathrm{mg} / \mathrm{kg} /$ day $)$ followed by oral itraconazole (400 mg/day). There was no consistent duration of antifungal therapy. Twelve patients received antifungal therapy, four of whom died during treatment. The other eight patients recovered, four of whom showed no recurrence, but the other four experienced a relapse 3-24 months later during follow-up. One of these patients received intravenous amphotericin B deoxycholate followed by itraconazole antifungal treatment for
18 months, but relapsed 6 months after discontinuing treatment. Antifungal treatment was discontinued in two patients because of severe multiple organ failure, and these patients subsequently died.

\section{Discussion}

Penicilliosis is a common but serious opportunistic fungal infection in AIDS patients in Southeast Asia, but is also increasingly observed in HIV-negative individuals. PSM disseminates hematogenously to other locations, with the reticuloendothelial system, skin, and lungs
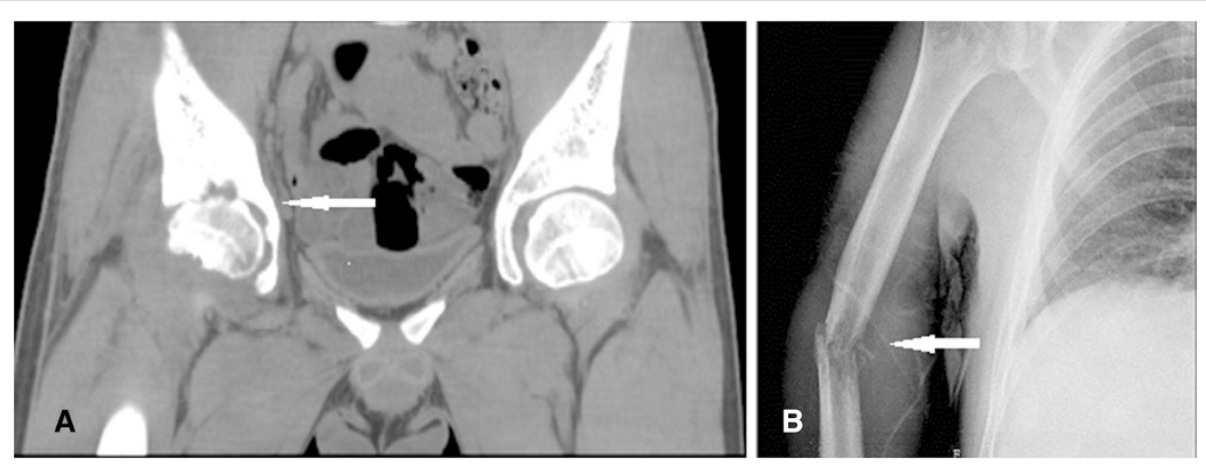

Figure 2 Radiography and computed tomography showing periosteal proliferation in the innominatum and femur (A), and a pathologic fracture $(B)$ in the humerus. 

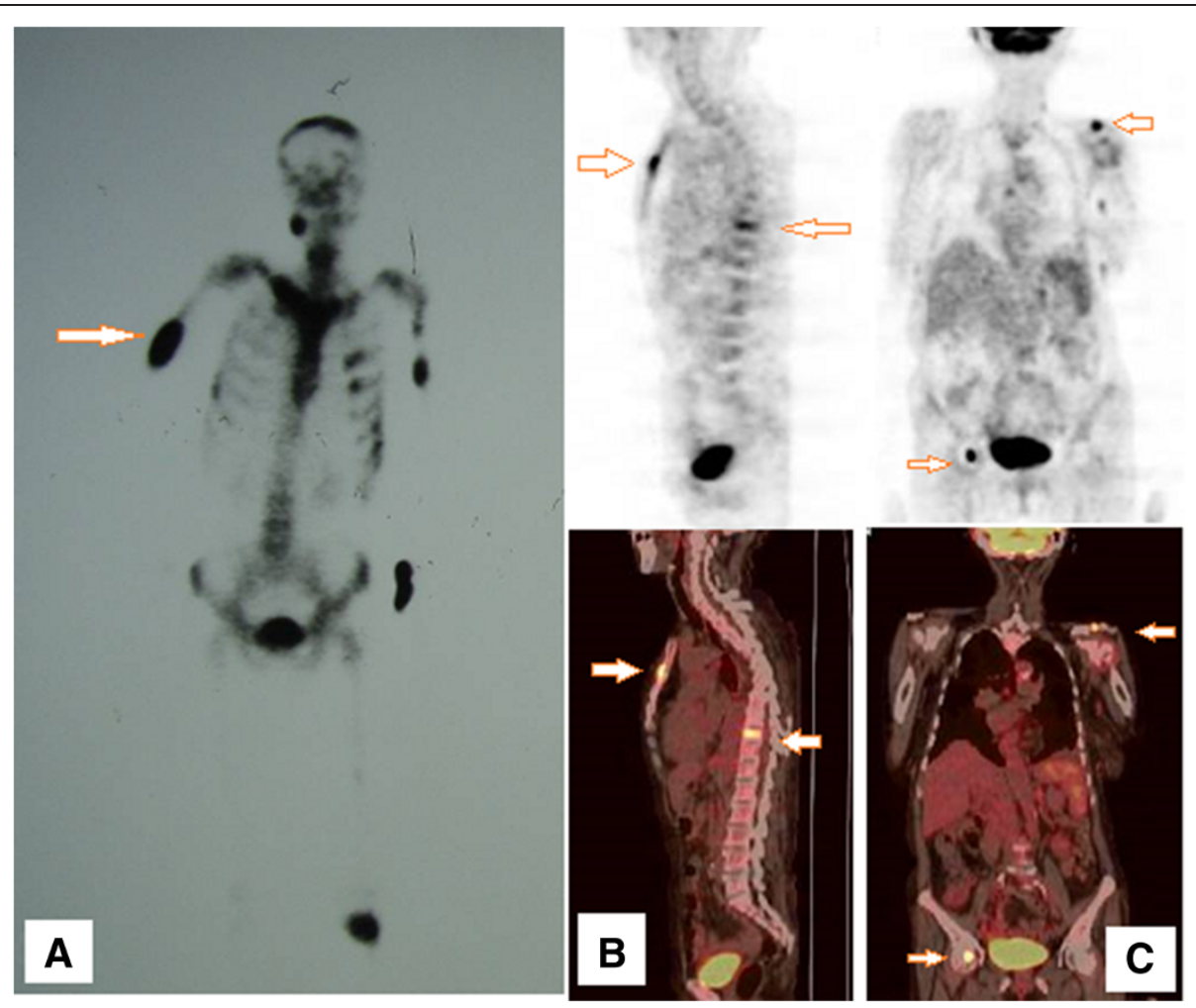

Figure 3 Emission computed tomography (CT) showing increased radioactive concentration in the brustwirbels, sternum, ilium, and left clavicle (A). Positron emission tomography/CT showed generalized lymphadenopathy, increased whole-body bone metabolic activity, and generalized skeletal destruction. The ${ }^{18}$ F-FDG standard uptake value was increased in the whole skeleton and was 5.1 in the sternum (B), and 6.1 in the femur and clavicle (C).

being the most commonly involved sites [6,2]. However, $P$. marneffei infection of the bone leading to osteolytic lesions is extremely rare [3].

In the current retrospective analysis, 100 patients were diagnosed with disseminated PSM, including 65 HIVinfected and 35 HIV-negative patients. All of the 14 patients with osteolytic lesions were HIV-negative, indicating a high incidence rate of PSM with osteolytic lesions of $40 \%$ in HIV-negative patients. The presence of $P$. marneffei infection with osteolytic lesions predominantly in HIVnegative patients was consistent with previous reports $[7,8]$. Thus, despite the importance of osteolytic lesions, they are easily overlooked in HIV-negative patients with P. marneffei infection.

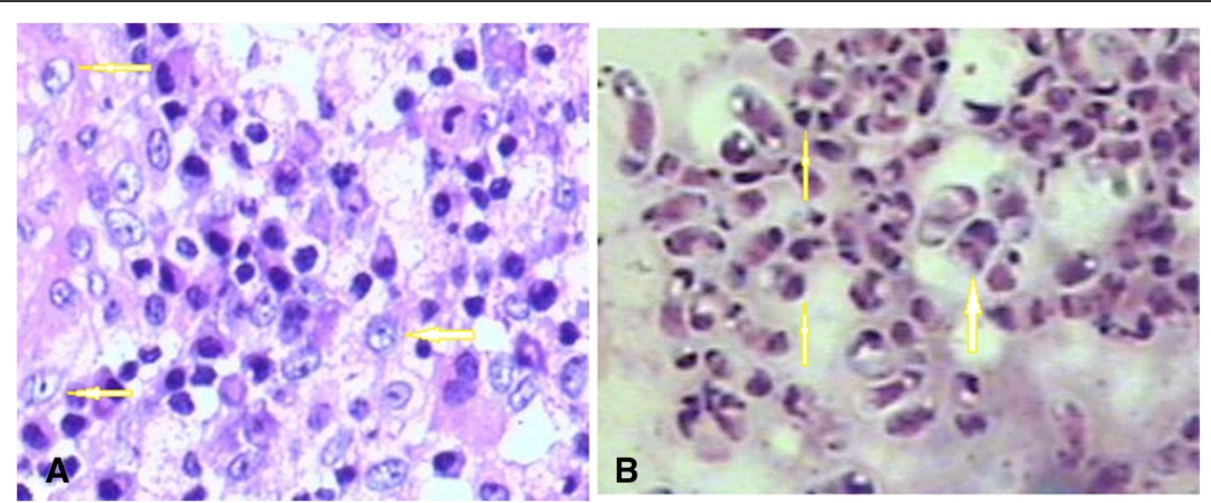

Figure 4 Histopathology of cervical vertebral (periodic acid-Schiff stain, 400x) (A) and bone marrow samples. Numerous intracellular yeast-like or sausage-like cells measuring 2-3 $\mathrm{m}$ in diameter with a transverse septum were observed (arrows, periodic acid-Schiff staining, 1000x) (B). 
Osteolytic lesions primarily occur in HIV-negative patients with $P$. marneffei infection because of their significantly higher white cell population and stronger neutrophil function compared with HIV-positive patients [6,7]. HIV-positive patients also have relatively higher serum antigen titers, while HIV-negative patients have higher serum antibody concentrations, as determined by $P$. marneffei-specific mannoprotein Mp1p ELISA [6]. In HIV-negative patients, when P. marneffei disseminates to an osseous site, the significant focal neutrophil aggregation results in localized granulomas and abscesses, leading to significantly increased uptake at osteolytic sites on bone ECT and increased wholebody bone metabolic activity on PET/CT. In contrast, neutrophil function is decreased in immunodeficient HIV-positive patients, and P. marneffei infection of osseous sites is therefore rare. There is a prevailing hypothesis that osteolytic lesions typically occur at sites of neutrophil accumulation, where the release of proteolytic enzymes leads to tissue lysis, liquefaction, and necrosis [3].

In our study, PSM with osteolytic lesions could involve any bone, including bones of the trunk, limbs, skull, and tracheal cartilage. The most commonly involved sites were the vertebrae, skull, femur, and ribs, followed by the ilium, clavicle, scapula, humerus, and tibia. The radius, sternum, innominatum, fibula, bones of the hand, humerus, ischium, pubis acetabulum, bones of the foot, sacrum, and cartilage were also involved. Most patients also presented with swelling and tenderness in the soft tissues and joints surrounding the bones. The most striking findings were multiple well-delineated osteolytic lesions, which indicated a predilection for flat bones and long bones, consistent with previous studies $[7,8]$.

The radiographic findings revealed well-circumscribed osteolytic lesions with multiple lucent defects showing a moth-eaten appearance, periosteal proliferation, and fracture. ECT showed significantly increased uptake in many parts of the skeleton, while PET/CT showed increased whole-body bone metabolic activity and diffuse skeletal destruction. ${ }^{18}$ F-FDG SUV was increased in the entire skeleton, with a mean ${ }^{18}$ F-FDG SUV of 6.16. Collectively, these findings resemble those of malignancy. Diagnosis is often delayed because penicilliosis marneffei is considered a rare condition in HIV-negative individuals. The radiographic findings usually lag behind the clinical findings by weeks or months, and the variable radiographic presentations and lesion distributions resemble those seen in osteomyelitis caused by tuberculosis, nontuberculous mycobacterial infection, tumors, and other fungal conditions such as African histoplasmosis, blastomycosis, cryptococcosis, and coccidioidomycosis [9-11]. In our study population, osteolytic lesions were a common but easily overlooked finding in HIV-negative individuals with P. marneffei infection. P. marneffei infection should therefore be considered as one of the common causative organisms in the differential diagnoses of fungal osteomyelitis.

Taking clinical symptoms and signs into consideration, P. marneffei osteomyelitis can be diagnosed by its radiological features, typical clinical features, histopathologic examination, and organism culture, specifically from bone, bone marrow, and skin lesions. Bone biopsy with image guidance can increase the positivity rate of fungal cultures.

Penicilliosis is very susceptible to antifungal treatment, and P. marneffei is susceptible to itraconazole and amphotericin B in vitro [12]. The recommended dosage and duration of treatment among HIV-infected patients are intravenous amphotericin $\mathrm{B}$ deoxycholate $0.6-1.0 \mathrm{mg} / \mathrm{kg} /$ day for 2 weeks, followed by oral itraconazole $400 \mathrm{mg} /$ day for 8-10 weeks [13]; however, there is currently no recommended treatment for HIVnegative individuals. Six patients died in the current study, representing a high mortality $(42.85 \%)$, while a further four patients relapsed 3-24 months later despite long-term antifungal treatment, representing a high recurrence rate $(28.57 \%)$. The patients in this study received antifungal treatment for a longer duration than individuals without osteolysis. There are currently no standard recommendations for the appropriate treatment duration and for prophylaxis of PSM with osteolysis, and treatment depends on the severity of the infection, the response to therapy, and the patient's immune status [1]. Surgery and external fixation should be performed in patients with persistent or refractory bone disease, such as pathologic fracture and pyogenic osteomyelitis. Our anecdotal experience revealed that relapse was more common in patients who received a shorter duration of treatment. P. marneffei involving the bone and leading to osteolysis thus demonstrates the potential of systemic infection to cause severe lesions, and to be associated with poor prognosis and a high recurrence rate, highlighting the need for prolonged antifungal treatment.

\section{Conclusions}

PSM disseminates hematogenously to other locations. However, $P$. marneffei infection of the bone leading to osteolytic lesions is extremely rare. Osteolysis is often overlooked in HIV-negative individuals with disseminated $P$. marneffei infection. However, P. marneffei involving the bone and leading to osteolysis may indicate severe systemic disturbance, and is characterized by a poor prognosis, high recurrence rate, and the need for prolonged antifungal treatment. 


\section{Consent}

Written informed consent was obtained from all the patients for publication of this report and any accompanying images. A copy of the written consent is available for review by the Editor of this journal.

\section{Competing interests}

The authors declare that they have no competing interests.

\section{Authors' contributions}

YQ made substantial contributions to the conception and design of the study; acquisition, analysis, and interpretation of the data; and drafting of the manuscript. JZ and GL made substantial contributions to the conception and design of the study; acquisition, analysis, and interpretation of the data; and critical revision of the manuscript for important intellectual content. JZ and GL also gave final approval of the version to be published and agree to be accountable for all aspects of the work in ensuring that questions related to the accuracy and integrity of any part of the work are appropriately investigated and resolved. XZ gave final approval of the version to be published. $\mathrm{ZH}$ participated in analysis and interpretation of the data and agrees to be accountable for all aspects of the work in ensuring that questions related to the accuracy or integrity of any part of the work are appropriately investigated and resolved. JD and JB conceived of the study, participated in its design, and helped to draft the manuscript. All authors read and approved the final manuscript.

\section{Authors' information}

Ye Qiu and Jianquan Zhang are co-first authors.

\section{Author details}

${ }^{1}$ Department of Integrated Medicine, The Affiliated Tumor Hospital of Guangxi Medical University, Nanning, Guangxi, China. ${ }^{2}$ Department of Respiratory Medicine, The First Affiliated Hospital of Guangxi Medical University, Nanning, Guangxi 530021, China.

Received: 23 July 2014 Accepted: 23 January 2015

Published online: 06 February 2015

\section{References}

1. Zhou F, Bi X, Zou X, Xu Z, Zhang T. Retrospective analysis of 15 Cases of Penicilliosis marneffei in a Southern China Hospital. Mycopathologia. 2014;177(5-6):271-9.

2. Chitasombat M, Supparatpinyo K. Penicillium marneffei infection in immunocompromised host. Curr Fungal Infect Rep. 2013;7:44-50.

3. Liu GN, Huang JS, Zhong XN, Zhang JQ, Zou ZX, Yang ML, et al. A Penicillium marneffei infection within an osteolytic lesion in a HIV-negative patient. Int J Infect Dis. 2014;23:1-3.

4. Duong TA. Infection due to Penicillium marneffei, an emerging3 pathogen: review of 155 reported cases. Clin Infect Dis. 1996;23:125-30.

5. Yousukh A, Jutavijittum $P$, Pisetpongsa $P$, Chitapanarux $T$, Thongsawat $S$, Senba M, et al. Clinicopathologic study of hepatic Penicillium marneffei in Northern Thailand. Arch Pathol Lab Med. 2004;128(2):191-4.

6. Zhang JQ, Yang ML, Zhong XN, He ZY, Liu GN, Deng JM, et al. A comparative analysis of the clinical and laboratory characteristics in disseminated penicilliosis marneffei in patients with and without human immunodeficiency virus infection. Zhonghua Jie He He Hu Xi Za Zhi. 2008;31:740-6.

7. Deng Z, Ribas JL, Gibson DW, Connor DH. Infections caused by Penicillium marneffei in China and Southeast Asia: review of eighteen published cases and report of four more Chinese cases. Rev Infect Dis. 1988;10:640-52.

8. Chan YF, Woo KC. Penicillium marneffei osteomyelitis. J Bone Joint Surg (Br). 1990;72:500-3.

9. Zhang Y, Yu YS, Tang ZH, Zang GQ. Cryptococcal osteomyelitis of the scapula and rib in an immunocompetent patient. Med Mycol. 2012;50:751-5.

10. Molter CM, Zuba JR, Papendick R. Cryptococcus gattii osteomyelitis and compounded itraconazole treatmentfailure in a Pesquet's parrot (Psittrichas fulgidus). J Zoo Wildl Med. 2014;45(1):127-33.

11. Schwarz J. What's new in mycotic bone and joint diseases. Pathol Res Pract. 1984;178:617-34
12. Qiu Y, Zhang J, Liu G, Zhong X, Deng J, He Z, et al. A case of penicillium marneffei infection involving the main tracheal structure. BMC Infect Dis. 2014;14:242.

13. Kawila R, Chaiwarith R, Supparatpinyo K. Clinical and laboratory characteristics of penicilliosis marneffei among patients with and without HIV infection in Northern Thailand: a retrospective study. BMC Infect Dis. 2013;13:464

\section{Submit your next manuscript to BioMed Central and take full advantage of:}

- Convenient online submission

- Thorough peer review

- No space constraints or color figure charges

- Immediate publication on acceptance

- Inclusion in PubMed, CAS, Scopus and Google Scholar

- Research which is freely available for redistribution 